\title{
Correction to: Evaluating author name disambiguation for digital libraries: a case of DBLP
}

\author{
Jinseok Kim ${ }^{1} \mathbb{D}$ \\ Published online: 27 November 2018 \\ (c) Akadémiai Kiadó, Budapest, Hungary 2018
}

\section{Correction to: Scientometrics (2018) 116:1867-1886 https://doi.org/10.1007/s11192-018-2824-5}

In the original publication of the article, in Abstract, the size of labeled data was incorrectly reported: "5000 to $6 \mathrm{M}$ disambiguated names" should be read as "5000 instances to $6 \mathrm{M}$ pairs of disambiguated names." Hence, the correct version in Abstract (4th sentence) should read as "...Tested on three types of labeled data containing 5000 instances to $6 \mathrm{M}$ pairs of disambiguated names, DBLP is shown to assign author names quite accurately to distinct authors, resulting in pairwise precision, recall, and F1 measures around 0.90 or above overall...".

Also, in Table 1, the number of uniquely identified authors was incorrectly reported for the study of Louppe et al. (2016): "36,340 authors" should be read as "15,388 authors." Hence, the correct version in Table 1 (last row, 4th column) should read as "360,066 paper records of 15,388 authors identified by original authors, curators, and publishers."

The original article can be found online at https://doi.org/10.1007/s11192-018-2824-5.

Jinseok Kim

jinseokk@umich.edu; jinseok.academic@gmail.com

1 Institute for Research on Innovation and Science, University of Michigan, 330 Packard Street, Ann Arbor, MI 48104-2910, USA 